\title{
Résumé de la mise à jour du CCNI sur I'utilisation recommandée du vaccin contre le virus du papillome humain (VPH) : Calendrier de vaccination du vaccin nonavalent contre le VPH à deux doses et utilisation des vaccins anti-VPH chez les populations immunodéprimées
}

\author{
SL Deeks ${ }^{1,2}, \mathrm{MC}$ Tunis $^{3}, \mathrm{~S}$ Ismail ${ }^{3}$ au nom du Comité consultatif national de l'immunisation (CCNI)*
}

\section{Résumé}

Contexte : Les infections par le virus du papillome humain (VPH) sont les infections transmissibles sexuellement les plus répandues. En l'absence de vaccination, on estime que $75 \%$ des Canadiens sexuellement actifs auront une infection au virus du papillome humain au cours de leur vie. Les programmes de vaccination contre le virus du papillome humain ont d'abord été recommandés par le Comité consultatif national de l'immunisation (CCNI) en 2007. En plus des options de vaccination contre le virus du papillome humain au Canada, le CCNI a récemment recommandé l'utilisation du vaccin nonavalent contre le virus du papillome humain (VPH9) nouvellement autorisé selon un calendrier de vaccination à trois doses pour la prévention des verrues et cancers anogénitaux associés au virus du papillome humain de types $6,11,16$ $18,31,33,45,52$ et 58 chez les filles et les femmes âgées de 9 à 45 ans et chez les garçons et les hommes âgés de 9 à 26 ans. II existe de nouvelles données qui évaluent un calendrier de vaccination à deux doses dans le cas du vaccin VPH9 chez les hommes et les femmes, que le CCNI a examiné en vue de fournir, en temps opportun, des conseils sur la possibilité d'adopter un calendrier de vaccination à deux doses dans le cas du vaccin VPH9. Récemment, un nombre croissant d'études ont également examiné en particulier les réponses de sous-groupes immunodéprimés aux vaccins contre le virus du papillome humain, ce qui a aussi déclenché une analyse documentaire et la formulation de recommandations du CCNI sur ce sujet.

Objectifs : Examiner les données probantes à l'appui d'un calendrier de vaccination à deux doses dans le cas du vaccin VPH9 et formuler des recommandations sur le calendrier de vaccination; et résumer les données probantes provenant d'une récente analyse documentaire du CCNI sur l'utilisation des vaccins contre le virus du papillome humain chez les populations immunodéprimées et formuler des recommandations sur l'utilisation du vaccin contre le virus du papillome humain au sein de ces groupes.

Méthodologie : Le groupe de travail sur le virus du papillome humain (GTVPH) du CCNI a examiné les résultats d'un essai clinique du vaccin VPH9 administré selon un calendrier de vaccination à deux doses chez les hommes et les femmes (protocole V503-010) et a procédé à un sur le sujet de l'immunisation contre le virus du papillome humain des populations immunodéprimées. L'examen de la documentation du $\mathrm{CCNI}$ et la déclaration du CCNI ont été publiées séparément.

Résultats : Une seule étude a fait l'objet d'un calendrier de vaccination à deux doses avec le vaccin VPH9, soit un essai randomisé contrôlé de bonne qualité commandité par un grand fabricant (protocole V503-010). Dans le contexte d'études portant sur d'autres vaccins contre le virus du papillome humain, le $\mathrm{CCNI}$ a considéré que cette étude constituait un corpus de données probantes suffisant pour étayer ses recommandations. À la suite d'une analyse documentaire exhaustive, 27 études ont été retenues pour faire l'objet d'une synthèse des données probantes comprenant notamment des rapports sur l'immunogénicité ou l'innocuité des vaccins, ou les deux, pour les populations immunodéprimées.

Conclusions : En se fondant sur les données probantes examinées, le CCNI a formulé de nouvelles recommandations concernant l'utilisation du vaccin VPH9 selon un calendrier de vaccination à deux doses à 0,6 à 12 mois chez les jeunes et a mis à jour la catégorie des données probantes concernant l'utilisation des vaccins contre le virus du papillome humain chez les populations immunodéprimées.

\section{Affiliations}

1 Président du Groupe de travail du CCNI sur le virus du papillome humain, Toronto (Ontario)

${ }^{2}$ Immunisation et maladies évitables par la vaccination, Santé publique Ontario, Toronto (Ontario)

${ }^{3}$ Centre de l'immunisation et des maladies respiratoires infectieuses, Agence de la santé publique du Canada, Ottawa (Ontario)

*Correspondance : naci-ccni@ phac-aspc.gc.ca
Citation proposée : Tunis MC, Deeks SL, Ismail S, au nom du Comité consultatif national de l'immunisation (CCNI). Résumé de la mise à jour du CCNI sur l'utilisation recommandée du vaccin contre le virus du papillome humain (VPH) : Calendrier de vaccination du vaccin nonavalent contre le VPH à deux doses et utilisation des vaccins anti-VPH chez les populations immunodéprimées. Relevé des maladies transmissibles au Canada. 2017;43(6):155-9. https://doi.org/10.14745/ccdr.v43i06a04f 


\section{Introduction}

Les infections au virus du papillome humain sont les infections transmissibles sexuellement les plus répandues. En l'absence de vaccination, on estime que $75 \%$ des Canadiens sexuellement actifs auront une infection à virus du papillome humain au cours de leur vie. Les vaccins quadrivalent (VPH4) et bivalent (VPH2) sont autorisés au Canada depuis 2006 et 2010, respectivement. Le Comité consultatif national de l'immunisation (CCNI) recommandait auparavant l'immunisation par le vaccin VPH4 chez les hommes et les femmes selon un calendrier à trois doses (à 0,2 et 6 mois) ou à deux doses (à 0 et 6 mois), ou par le vaccin VPH2 chez les femmes selon un calendrier à trois doses (à 0,1 et 6 mois) ou à deux doses (à 0 et 6 mois), en fonction de l'âge et de l'état de santé du sujet vacciné. En février 2015, un vaccin nonavalent contre le virus du papillome humain (VPH9) (Gardasil ${ }^{\circledR 9}$, Merck Canada, Inc.) a été autorisé au Canada pour la prévention des verrues et cancers anogénitaux associés au virus du papillome humain de types $6,11,16,18,31,33,45,52$ et 58 chez les filles et les femmes âgées de 9 à 45 ans et chez les garçons et les hommes âgés de 9 à 26 ans; ce vaccin a été recommandé par le $\mathrm{CCNI}$ selon un calendrier de vaccination à trois doses dans une déclaration du CCNI (1) et dans le résumé (2) du Relevé des maladies transmissibles au Canada (RMTC) publié en juillet 2016. L'immunisation contre les types 16 et 18 du virus du papillome humain peut prévenir environ $70 \%$ des cancers anogénitaux et $60 \%$ des lésions précancéreuses du col de l'utérus à risque élevé. On estime que l'immunisation contre les cinq autres types de virus du papillome humain contenus dans le vaccin VPH9 peut prévenir jusqu'à $14 \%$ de plus de cancers anogénitaux et jusqu'à $30 \%$ des lésions précancéreuses du col de l'utérus à risque élevé.

Le CCNI a passé en revue les nouvelles données probantes d'essais cliniques d'un calendrier de vaccination à deux doses du vaccin VPH9 en vue de fournir, en temps opportun, des conseils sur la possibilité d'adopter un calendrier de vaccination à deux doses du vaccin VPH9. Les déclarations précédentes du CCNI traitaient également de l'utilisation des vaccins contre le virus du papillome humain chez les populations immunodéprimées, mais cela s'est fait largement en l'absence de preuves solides pour ces groupes. Récemment, un nombre croissant d'études ont particulièrement examiné les réponses de sous-groupes immunodéprimés aux vaccins contre le virus du papillome humain, ce qui a déclenché une analyse documentaire du CCNI sur ce sujet et la formulation de recommandations. Le présent article résume la récente déclaration du CCNI sur les données probantes et les recommandations relatives à un calendrier de vaccination à deux doses du vaccin VPH9 (3) et l'examen de la documentation du CCNI qui met à jour les données probantes sur l'utilisation des vaccins contre le virus du papillome humain chez les personnes immunodéprimées (4). Ces nouvelles recommandations sont publiées dans la déclaration complète du CCNI (3) et également dans le chapitre sur le virus du papillome humain du Guide canadien d'immunisation, où toutes les recommandations courantes du CCNI sur le virus du papillome humain sont résumées et regroupées (5).

\section{Méthodologie}

Le CCNI a examiné les questions clés relatives au vaccin VPH9 proposées par le GTVPH , à savoir notamment : I'innocuité, l'immunogénicité et l'efficacité du vaccin VPH9 selon un calendrier de vaccination à deux doses. Un seul essai clinique commandité par un fabricant a été cerné (protocole V503-010) (6). Le GTVPH du CCNI a examiné les méthodes et les résultats de l'étude détaillés fournis par le fabricant du vaccin. Deux examinateurs indépendants ont évalué la validité interne de l'étude afin de déterminer le niveau et la qualité des données probantes, évaluation validée par le président du groupe de travail, et l'étude a fait l'objet d'un processus d'examen par les pairs modifié par le GTVPH et le CCNI.

Le CCNI a également examiné diverses questions clés en vue d'une analyse documentaire sur les vaccins contre le VPH chez les populations immunodéprimées, comme l'a proposé le GTVPH , y compris la question précise suivante : "Les résultats de la vaccination sont-ils différents d'un sous-groupe de populations immunodéprimées à l'autre? ». Une analyse documentaire a été effectuée selon la méthodologie du CCNI établie (7). Les détails de cette analyse documentaire et de la méthodologie connexe (termes de recherche, critères d'inclusion/d'exclusion, bases de données, nombre de résultats) se trouvent dans l'Examen de la documentation du CCNI concernant l'immunisation contre le VPH des populations immunodéprimées (4). Les recommandations proposées ont été élaborées à la suite d'un examen de la documentation. Le CCNI a approuvé des recommandations précises fondées sur des données probantes et a clarifié le raisonnement et les considérations pertinentes dans la mise à jour de la déclaration.

\section{Résultats}

Un essai randomisé contrôlé à grande échelle de bonne qualité a démontré qu'en suivant un calendrier de vaccination à deux doses chez les filles et les garçons de 9 à 14 ans, l'immunogénicité était non inférieure à celle d'un calendrier à trois doses chez les femmes de 16 à 26 ans. Dans le contexte d'études sur les calendriers de vaccination à deux doses $\mathrm{d}^{\prime}$ autres vaccins contre le virus du papillome humain, le CCNI a considéré le corpus de données probantes suffisant pour étayer ses recommandations relatives à un calendrier de vaccination à deux doses du vaccin VPH9.

En ce qui concerne les populations immunodéprimées, l'examen documentaire a recensé 27 études de synthèse de données probantes, notamment des rapports sur l'immunogénicité, I'innocuité ou les deux (4). Les études ont été regroupées en deux grandes catégories : les populations infectées au VIH et les groupes soumis à une immunosuppression thérapeutique. L'étude de cohorte était la conception expérimentale prédominante et la plupart de ces études comprenaient un faible nombre de participants et la qualité variait de bonne à mauvaise.

Des schémas immunosuppresseurs ont généralement été signalés chez les patients atteints d'immunodéficience thérapeutique acquise, mais malheureusement, ces études n'ont pas souvent stratifié les résultats en fonction de la modalité de traitement. Le degré d'immunosuppression efficace n'a pas été mesuré ou signalé dans aucune étude, mais la numération des lymphocytes CD4 était généralement indiquée dans le cas des études portant sur des patients infectés au VIH. Toutes les études ont utilisé des vaccins VPH2 ou VPH4 contre le virus du papillome humain en trois doses administrées à intervalles réguliers, à l'exception d'une étude, dans le cadre de laquelle une quatrième dose supplémentaire a été administrée. Aucune étude n'a utilisé le vaccin VPH9 autorisé récemment. 


\section{Conclusion}

En se fondant sur les données probantes examinées, le $\mathrm{CCNI}$ a publié trois recommandations sur l'utilisation d'un calendrier de vaccination à deux doses chez les populations immunocompétentes, et l'utilisation de vaccins contre le virus du papillome humain chez les populations immunodéprimées (encadré).

\title{
RECOMMANDATIONS CONCERNANT LE CALENDRIER DE VACCINATION AU VPH9 À DEUX DOSES CHEZ LES POPULATIONS IMMUNOCOMPÉTENTES
}

\author{
Recommandation n 1 - Filles et garçons immunocompétents de 9 à 14 ans \\ Le CCNI recommande que le vaccin VPH9 soit offert aux filles et aux garçons immunocompétents de 9 à 14 ans selon un calendrier de \\ vaccination à deux ou trois doses (comme c'est le cas des vaccins VPH2 ou VPH4 chez les filles et du vaccin VPH4 chez les garçons de cette \\ population) - Recommandation du CCNI selon les données probantes de catégorie B (données probantes acceptables pour recommander \\ la vaccination) \\ Le CCNI conclut qu'il y a dorénavant des données probantes acceptables pour recommander un calendrier de vaccination à deux doses dans le \\ cas du vaccin VPH9, bien que les faits probants soient limités en quantité. Par conséquent, en s'appuyant sur les données actuelles examinées \\ en vue de la présente déclaration et dans le cadre des déclarations précédentes du comité consultatif, le CCNI a conclu qu'il existe des données \\ acceptables pour recommander un calendrier de vaccination à deux ou à trois doses du vaccin VPH9 (données probantes de catégorie B) et \\ qu'il existe des données probantes de grande qualité pour recommander un calendrier de vaccination à deux ou à trois doses des vaccins \\ VPH2 ou VPH4 (chez les filles) et du vaccin VPH4 (chez les garçons). Dans un calendrier de vaccination contre le VPH à deux doses de n'importe \\ quel vaccin contre le VPH dont l'utilisation est autorisée au Canada, la seconde dose ne devrait pas être administrée plus tôt que 24 semaines \\ (6 mois) après la première dose. Bien que les données de suivi à long terme ne soient pas encore disponibles pour un calendrier de vaccination \\ au VPH9 à deux doses, une étude visant à évaluer la durée des réponses aux vaccins jusqu'à 36 mois est en cours. Au fur et à mesure que de \\ nouvelles données probantes deviendront disponibles, la catégorie de cette recommandation pourrait changer. II n'existe aucune donnée \\ probante indiquant que les personnes répondront différemment au vaccin VPH9, comparativement aux vaccins VPH2 ou VPH4.
}

\section{Recommandation $n^{\circ} 2$ - Femmes et hommes immunocompétents de 15 ans et plus}

Le CCNI continue de recommander que le vaccin VPH9 soit offert aux femmes et aux hommes immunocompétents de 15 ans et plus selon un calendrier de vaccination à trois doses (comme c'est le cas des vaccins VPH2 ou VPH4 chez les femmes et du vaccin VPH4 chez les hommes) - Recommandation du CCNI selon les données probantes de catégorie B (données probantes acceptables pour recommander la vaccination)

II n'existe pas d'étude évaluant directement un calendrier de vaccination à deux doses pour ce qui est du vaccin VPH9 chez les femmes et les hommes de 15 ans et plus. Par conséquent, un calendrier à trois doses continue de leur être recommandé. Cette recommandation est décrite dans la déclaration précédente intitulée «Recommandations mises à jour sur les vaccins contre le virus du papillome humain (VPH) : vaccin nonavalent contre le VPH et précisions sur les intervalles minimaux entre les doses dans le calendrier de vaccination contre le VPH ». Cependant, une récente étude réalisée en Inde laisse entendre que l'administration de deux doses du vaccin VPH4 peut être immunogène chez les jeunes filles de 10 à 18 ans; le CCNI continuera donc d'examiner les données probantes semblables au fur et à mesure qu'elles deviendront disponibles afin de déterminer le calendrier de vaccination optimal au VPH9 pour les personnes de 15 ans et plus.

\section{RECOMMANDATIONS CONCERNANT LA VACCINATION CONTRE LE VPH CHEZ LES PERSONNES IMMUNODÉPRIMÉES EN RAISON D'UNE MALADIE OU DE MÉDICAMENTS}

\section{Recommandation $n^{\circ} 3$}

Le CCNI continue de recommander que les vaccins contre le VPH soient administrés selon un calendrier à trois doses chez les populations immunodéprimées conformément aux lignes directrices actuelles liées à l'âge - Recommandation du CCNI selon les données probantes de catégorie B en ce qui a trait aux vaccins VPH2 et VPH4; (données probantes acceptables pour recommander la vaccination); Recommandations du CCNI selon les données probantes de catégorie I concernant le vaccin VPH9 (données probantes insuffisantes, en quantité ou en qualité, pour formuler une recommandation; cependant, d'autres facteurs peuvent influer sur la prise de décisions)

Bien que les recommandations du CCNI concernant les vaccins VPH2 et VPH4 chez cette population demeurent inchangées, en s'appuyant sur l'analyse documentaire menée afin d'éclairer ces recommandations, le CCNI a réajusté à la hausse la catégorie des données probantes (fondant les recommandations), qui passe ainsi de la catégorie I (données probantes insuffisantes, en quantité ou en qualité, pour formuler une recommandation; d'autres facteurs pouvant toutefois influer sur la prise de décisions) à la catégorie B (données probantes acceptables pour formuler une recommandation). Les données probantes ne laissent pas présager de préoccupations particulières en matière d'innocuité relativement à I'utilisation des vaccins VPH2 ou VPH4 chez les populations immunodéprimées. À l'heure actuelle, il n'existe pas d'étude évaluant directement l'immunogénicité, l'efficacité ou l'innocuité du vaccin VPH9 chez les populations immunodéprimées selon un calendrier de vaccination à trois doses ou à deux doses (par conséquent, la catégorie des données probantes sur lesquelles les recommandations sont fondées est la catégorie I). Toutefois, il n'existe aucune donnée probante indiquant que les personnes répondraient différemment au vaccin VPH9, comparativement aux vaccins VPH2 ou VPH4. Au fur et à mesure que de nouvelles données probantes deviendront disponibles, la catégorie de cette recommandation pourrait changer.

II n'y a actuellement aucune étude publiée qui se penche sur l'utilisation d'un calendrier de vaccination contre le VPH à deux doses chez les populations immunodéprimées. Le CCNI conclut qu'il existe des données probantes acceptables démontrant que l'immunogénicité des vaccins VPH2 et VPH4 peut être diminuée chez les populations immunodéprimées à la suite d'un calendrier de vaccination à trois doses, bien que les réponses des anticorps dépassent toujours généralement celles découlant d'une infection naturelle chez les personnes immunocompétentes. Par conséquent, bien que l'immunogénicité et l'efficacité n'aient pas été entièrement caractérisées dans toutes les populations immunodéprimées, on s'attend à ce que les personnes immunodéprimées tirent profit de ces vaccins; le CCNI continue donc de recommander la vaccination chez ces groupes en utilisant un calendrier à trois doses pour assurer leur protection. 
Le tableau 1 résume les recommandations énoncées ci-dessus selon le calendrier de vaccination approprié et la population à laquelle le vaccin contre le virus du papillome humain est administré. L'immunisation contre le VPH peut être complétée avec le vaccin VPH2, VPH4 ou VPH9 chez les femmes et avec les vaccins VPH4 ou VPH9 chez les hommes, selon les calendriers de vaccination résumés dans ce tableau. Dans la mesure du possible, le même vaccin doit être utilisé pour compléter la série de vaccins. Si l'administration de la série vaccinale avec le même vaccin n'est pas possible, les vaccins VPH2, VPH4 ou VPH9 peuvent être utilisés pour compléter la série chez les femmes, et les vaccins VPH4 ou VPH9 peuvent être utilisés pour compléter la série chez les hommes. Le vaccin VPH9 administré à des personnes immunocompétentes âgées de 9 à 26 ans devrait offrir une protection d'efficacité semblable contre les génotypes contenus dans le vaccin VPH4. En outre, le vaccin VPH9 offre une protection contre les cinq types supplémentaires du virus du papillome humain non contenus dans le vaccin VPH4 (types 31, $33,45,52$ et 58 du virus du papillome humain).

Tableau 1 : Recommandations du CCNI concernant le calendrier d'immunisation contre le virus du papillome humain

\begin{tabular}{|c|c|c|}
\hline $\begin{array}{l}\text { Groupes } \\
\text { recommandés }\end{array}$ & $\begin{array}{l}\text { Calendrier } \\
\text { d'immunisation } \\
\text { recommandé }\end{array}$ & $\begin{array}{l}\text { Vaccins contre } \\
\text { le virus du } \\
\text { papillome humain } \\
\text { et catégorie } \\
\text { de données } \\
\text { probantes du } \\
\text { CCNI (7) }\end{array}$ \\
\hline $\begin{array}{l}\text { Filles en santé } \\
\text { (immunocompétentes et } \\
\text { non infectées par le VIH) } \\
\text { âgées de } 9 \text { à } 14 \text { ans (et } \\
\text { femmes en santé âgées } \\
\text { de } 15 \text { ans et plus chez qui } \\
\text { la première dose a été } \\
\text { administrée entre } \\
9 \text { et } 14 \text { ans) }\end{array}$ & $\begin{array}{l}\text { Calendrier à deux } \\
\text { ou à trois doses }\end{array}$ & $\begin{array}{l}\text { VPH2 ou VPH4 } \\
\text { (catégorie A) } \\
\text { VPH9 (catégorie B) }\end{array}$ \\
\hline $\begin{array}{l}\text { Femmes en santé } \\
\text { âgées de } 15 \text { ans et plus } \\
\text { (immunocompétentes et } \\
\text { non infectées par le VIH) }\end{array}$ & $\begin{array}{l}\text { Calendrier à } \\
\text { trois doses }\end{array}$ & $\begin{array}{l}\text { VPH2 ou VPH4 } \\
\text { (catégorie A) ou } \\
\text { VPH9 (catégorie B) }\end{array}$ \\
\hline $\begin{array}{l}\text { Garçons en santé } \\
\text { (immunocompétents et non } \\
\text { infectés par le VIH) âgés } \\
\text { de } 9 \text { à } 14 \text { ans (et hommes } \\
\text { en santé âgés de } 15 \text { ans et } \\
\text { plus chez qui la première } \\
\text { dose a été administrée } \\
\text { entre } \\
9 \text { et } 14 \text { ans) }\end{array}$ & $\begin{array}{l}\text { Calendrier à deux } \\
\text { ou trois doses }\end{array}$ & $\begin{array}{l}\text { VPH4 ou } \\
\text { VPH9 (catégorie B) }\end{array}$ \\
\hline $\begin{array}{l}\text { Hommes en santé } \\
\text { âgés de } 15 \text { ans et plus } \\
\text { (immunocompétents et non } \\
\text { infectés par le VIH) }\end{array}$ & $\begin{array}{l}\text { Calendrier à } \\
\text { trois doses }\end{array}$ & $\begin{array}{l}\text { VPH4 ou } \\
\text { VPH9 (catégorie B) }\end{array}$ \\
\hline $\begin{array}{l}\text { Personnes } \\
\text { immunodéprimées } \\
\text { et personnes } \\
\text { immunocompétentes } \\
\text { infectées par le VIH }\end{array}$ & $\begin{array}{l}\text { Calendrier à } \\
\text { trois doses }\end{array}$ & $\begin{array}{l}\text { VPH2 ou VPH4 chez } \\
\text { les femmes (catégorie } \\
\text { B); } \\
\text { VPH4 chez hommes } \\
\text { (catégorie B); } \\
\text { VPH9 chez les femmes } \\
\text { ou les hommes } \\
\text { (catégorie I) }\end{array}$ \\
\hline
\end{tabular}

Des efforts devraient être faits pour administrer les vaccins contre le virus du papillome humain aux intervalles recommandés. Lorsqu'un calendrier abrégé est nécessaire, les intervalles minimaux entre les doses de vaccin doivent être respectés. Dans un calendrier à trois doses, I'intervalle minimal entre la première et la deuxième dose du vaccin est de quatre semaines, l'intervalle minimal entre la deuxième et la troisième dose du vaccin est de douze semaines et l'intervalle minimal entre la première et la dernière dose dans un calendrier à deux ou à trois doses est de 24 semaines.

\section{Déclaration des auteurs}

La présente déclaration a été préparée par le $D^{r}$. M. Tunis, la $D^{r e} S$. Ismail, la $D^{r e} S$. Deeks et a été approuvée par le CCNI.

\section{Conflit d'intérêt}

Aucun.

\section{Remerciements}

Membres du groupe de travail sur le virus du papillome humain : $D^{\text {re }}$ S. Deeks (présidente), D ${ }^{\text {re }}$ E. Castillo, Dr S. Dobson, $D^{r}$ M. Krajden, $D^{r e}$ G. Ogilvie et $D^{r e}$ C. Sauvageau

Membres du CCNI : Dr I. Gemmill (président), D ${ }^{\text {re }}$ C. Quach

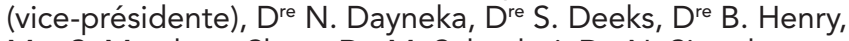
$M^{\text {me }}$ S. Marchant-Short, Dre M. Salvadori, Dre N. Sicard, $D^{r e}$ W. Vaudry, $D^{r} D$. Vinh et $D^{r}$ R. Warrington

Agents de liaison : $D^{r e} \mathrm{~J}$. Blake (Société des obstétriciens et gynécologues du Canada), Dr J. Brophy (Association canadienne pour la recherche et l'évaluation en immunisation [ACREI]), $D^{\text {re }}$ A. Cohn (Centers for Disease Control and Prevention, États-Unis), D ${ }^{\text {re }}$ J. Emili (Collège des médecins de famille du Canada), Dr M. Lavoie (Conseil des médecins hygiénistes en chef), Dre C. Mah (Association canadienne de santé publique), $D^{r e} D$. Moore (Société canadienne de pédiatrie), $D^{r e} A$. Pham-Huy (Association pour la microbiologie médicale et l'infectiologie Canada [AMMI]) et $\mathrm{M}^{\mathrm{me}} \mathrm{T}$. Coles (Comité canadien sur l'immunisation)

Anciens représentants de liaison : $\mathrm{M}^{\mathrm{me}} \mathrm{E}$. Sartison (Comité canadien sur l'immunisation)

Représentants d'office : $D^{\text {re }}$ (Capc) K. Barnes (Défense nationale et Forces armées canadiennes), $\mathrm{M}^{\mathrm{me}} \mathrm{G}$. Charos (Centre de I'immunisation et des maladies respiratoires infectieuses, Agence de la santé publique du Canada), D ${ }^{r e} \mathrm{G}$. Coleman (Direction des produits biologiques et des thérapies génétiques, Santé Canada), Dr J. Gallivan (Direction des produits de santé commercialisés, Santé Canada), Mme J. Pennock (Centre de l'immunisation et des maladies respiratoires infectieuses, Agence de la santé publique du Canada), $D^{r} \mathrm{~T}$. Wong (Direction générale de la santé des Premières nations et des Inuits, Santé Canada)

Le $\mathrm{CCNI}$ tient à souligner la contribution de $\mathrm{M}^{\text {me }} \mathrm{C}$. Jensen (Centre de l'immunisation et des maladies respiratoires infectieuses, Agence de la santé publique du Canada), de 
M. J. Nkanza (Centre de l'immunisation et des maladies respiratoires infectieuses, Agence de la santé publique du Canada) et de $\mathrm{M}^{\mathrm{me}} \mathrm{C}$. Smalley (Bibliothèque de la santé, Santé Canada).

\section{Financement}

Le travail du Comité consultatif national de l'immunisation a été appuyé par l'Agence de la santé publique du Canada.

\section{Références}

1. Gouvernement of Canada [Internet]. Recommandations mises à jour sur les vaccins contre le virus du papillome humain (VPH) : vaccine nonavalent contre le VPH et précisions sur les intervalles minimums entre les doses dans le calendrier d'immunisation contre le VPH. Ottawa: CCNI; 2016 [consulté le 28 juin 2016; consulté le 21 mars 2017]. Disponible à l'adresse : http://www.canadiensensante.gc.ca/ publications/healthy-living-vie-saine/human-papillomavirus9-valent-vaccine-update-recommendation-misesa-jour-recommandations-papillome-humain-vaccin-nona valent/index-fra.php.

2. Tunis MC, Deeks SL au nom du Comité consultatif national de l'immunisation (CCNI). Résumé des recommandations mises à jour du Comité consultatif national de l'immunisation sur les vaccins contre le virus du papillome humain (VPH) : Intervalles minimaux entres les doses du vaccin nonavalent contre le virus du papillome humain dans le calendrier d'immunisation contre le VPH. Relevé des maladies transmissible au Canada. 2016;42(7):166-9. Disponible à l'adresse : http://www.phac-aspc.gc.ca/publicat/ccdrrmtc/16vol42/dr-rm42-7/ar-03-fra.php.

3. Gouvernement du Canada [Internet]. Recommandations mises à jour du sur les vaccins contre le virus du papillome humain (VPH) : Calendrier de vaccination du vaccin nonavalent contre le VPH à deux doses et utilisation des vaccins anti-VPH chez les populations immunodéprimées Ottawa: CCNI; 2017 [mise à jour le 29 mai 2017] Disponible à l'adresse : https://www.canada.ca/fr/sante-publique/ services/publications/vie-saine/mises-a-jour-vaccins-contrevirus-papillome-humain-calendrier-vaccination-populationsimmunodeprimees.html.

4. Gouvernement du Canada [Internet]. Examen de la documentation du CCNI concernant l'immunisation contre le VPH des populations immunodéprimées. Ottawa: CCNI; 2017 [mise à jour le 29 mai 2017] Disponible à I'adresse : https://www.canada.ca/fr/sante-publique/ services/publications/vie-saine/examen-documentationimmunisation-contre-virus-papillome-humain-populationsimmunodeprimees.html.

5. Comité consultatif national de l'immunisation. Guide canadien d'immunisation: Partie 4 : Vaccin contre le virus du papillome humain. Ottawa (ON): Agence de la santé publique du Canada [mise à jour le 29 mai 2017]. Disponible à l'adresse : https://www.canada.ca/en/public-health/ services/publications/healthy-living/canadian-immunizationguide-part-4-active-vaccines/page-9-human-papillomavirusvaccine.html.

6. ClinicalTrials.gov [Internet]. Merck Sharp \& Dohme Corp. A Phase III Study of a 2-dose Regimen of a Multivalent Human Papillomavirus (HPV) Vaccine (V503), Administered to 9 to 14 Year-olds and Compared to Young Women, 10 to 26 Years Old (V503-101). [mise à jour le 9 mars 2017; consulté le 21 mars 2017]. Disponible à l'adresse : https://clinicaltrials.gov/ ct2/show/study/NCT01984697.

7. Agence de la santé publique du Canada [Internet]. Recommandations pour l'immunisation fondées sur des données probants - Méthodes du Comité consultatif national de l'immunisation. Ottawa: CCNI; 2009 [mise à jour le 2 fév 2009; consulté le 21 mars 2017]. Disponible à l'adresse : http://www.phac-aspc.gc.ca/publicat/ccdr-rmtc/09vol35/acs1/index-fra.php.

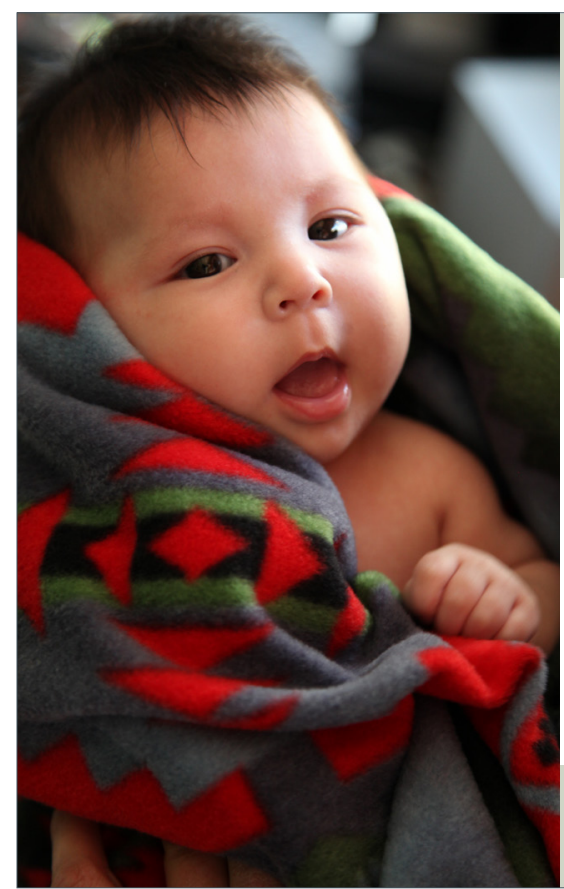

\title{
Des recherches sont en cours afin de mettre au point un nouveau vaccin pour un agent pathogène émergent dans nos collectivités.
}

\author{
Apprenez-en davantage dans le numéro \\ du mois de mai 2017 du RMTC : \\ Science de la mise en oeuvre
}

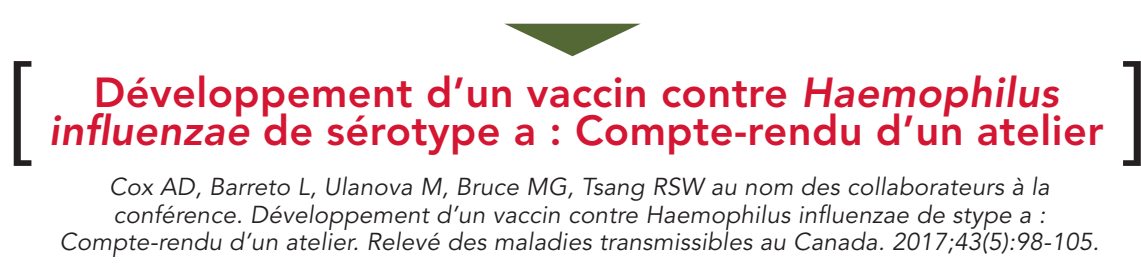

Obtenir le numéro complet :

Recherche Web "RMTC+Science de la mise en oeuvre" 